\title{
La relación de trabajo de retroceso de un ciclo Brayton
}

\section{Back Work Ratio of Brayton Cycle}

\author{
Malaver de la Fuente M. \\ Universidad Maritima del Caribe, Venezuela \\ E-mail:mmf_umc@hotmail.com
}

(Recibido: junio de 2008; reevaluado: junio de 2009; aceptado: diciembre de 2009)

\begin{abstract}
Resumen
En este artículo se estudia la relación que existe entre las temperaturas, la relación de trabajo de retroceso y el trabajo neto en el ciclo Brayton, que es el ciclo ideal que describe el comportamiento de los motores de turbina de gas. La aplicación de programas computarizados ayuda a mostrar la influencia de la relación de trabajo de retroceso o relación de acoplamiento, la temperatura de entrada al compresor y la temperatura de entrada a la turbina en este ciclo termodinámico ideal. Los resultados obtenidos permiten deducir que el valor máximo que alcanza la relación de trabajo de retroceso dependerá de los límites de temperatura máxima y mínima impuestos en el ciclo Brayton.
\end{abstract}

Descriptores: ciclo Brayton, ciclo termodinámico, compresor, relación de trabajo de retroceso, turbina de gas, trabajo neto.

\section{Abstract}

This paper analizes the existing relation between temperatures, back work ratio and net work of Brayton cycle, a cycle that describes gas turbine engines performance. The application of computational software helps to show the influence of back work ratio or coupling ratio, compressor and turbine inlet temperatures in an ideal thermodynamical cycle. The results lead to deduce that the maximum value reached in back work ratio will depend on the ranges of maximum and minimal temperatures of Brayton cycle.

Keywords: Brayton cycle, thermodynamical cycle, compressor, work retrocession relation, turbine gas, net work.

\section{Introducción}

En termodinámica existen dos áreas fundamentales de aplicación, la generación de potencia y la refrigeración. Su estudio se hace a través de ciclos termodinámicos, los cuales se dividen en ciclos de potencia y de refrigeración, entre los que se incluyen el ciclo de Carnot, Otto, Diesel, Dual, Rankine y Brayton (Cengel y Boles, 2006; Van Wylen et al., 2004; Wark y Richards, 2001).
Los ciclos termodinámicos se pueden clasificar en ciclos cerrados y abiertos. En los ciclos cerrados la sustancia de trabajo se regresa a su estado inicial al final del ciclo y debe recircularse. Los motores de combustión interna llamados también máquinas térmicas, funcionan de tal manera que los gases de combustión se escapan y se reemplazan por una nueva mezcla aire-combustible al terminar cada ciclo.

La operación estándar de los motores de combustión interna se realiza en el modo de presión limitada, o 
en el modo de volumen limitado (Russel y Adebiyi, 1997). Las turbinas de gas son un ejemplo del primer modo mientras que los motores de combustión interna como el motor Otto y Diesel se diseñan para funcionar en el modo de volumen limitado (Cengel y Boles, 2006; Russel y Adebiyi, 1997).

Los ciclos de potencia han sido objeto de valiosas idealizaciones que han sido útiles para el diseño (Herrera et al., 2008; Malaver, 2008; Cengel y Boles, 2006; Urrecheaga y Malaver, 2003; Wark y Richards, 2001; Guzmán y Angulo, 1998; Jones y Dugan, 1997; Leff, 1987; Curzon y Ahlborn, 1975), aún cuando las plantas se desvían de estas simplificaciones, porque máquinas y procesos reales son imperfectos y no se rigen fielmente por los esquemas ideales. Es por esta razón que modelar un sistema que trate de reproducir la realidad constituye un reto para la ingeniería, siempre expuesta a discusiones por las suposiciones y aproximaciones que se incorporan (Herrera et al., 2008 y Burghardt, 1984).

El análisis de los ciclos reales de los motores de combustión interna es complicado, debido a la presencia de fenómenos e irreversibilidades como la fricción y la rapidez de los procesos de expansión-compresión, que impiden establecer condiciones de equilibrio termodinámico; por lo que si se quiere hacer manejable el estudio de un ciclo se deben hacer algunas simplificaciones (Cengel y Boles, 2006).

Cuando a un ciclo real se le eliminan todos los problemas internos complejos, se obtiene un ciclo constituido por una secuencia de pasos reversibles. Un ciclo que cumple con estas condiciones recibe el nombre de ciclo ideal. Para una turbina de gas, el ciclo ideal es el ciclo Brayton.

\section{El ciclo de Brayton para una Turbina de gas}

El ciclo Brayton fue propuesto inicialmente por George Brayton al emplearlo en un motor que quemaba aceite, el cual diseñó personalmente en 1870 (Cengel y Boles, 2006; Jones y Dugan,1997). Las turbinas de gas usualmente operan en un ciclo abierto, como se ilustra en la figura 1. El aire en condiciones ambientales se introduce en un compresor axial o centrífugo donde su temperatura y presión se eleva. El aire de alta presión sigue hacia la cámara de combustión donde el combustible se quema a presión constante y los gases de alta temperatura que resultan entran a la turbina en donde se expanden hasta la presión atmosférica, de manera tal que producen potencia. Los gases de escape que salen de la turbina se expulsan hacia fuera (Dugan y Jones, 1997).

1. La sustancia de trabajo es un fluido que circula de modo continuo en un circuito cerrado y se comporta como un gas ideal.

2. Todos los procesos que integran el ciclo son reversibles.

3. El proceso de combustión se reemplaza por un proceso de adición térmica desde una fuente externa.

4. El proceso de escape se sustituye por un proceso de eliminación térmica que regresa la sustancia de trabajo a su estado inicial.

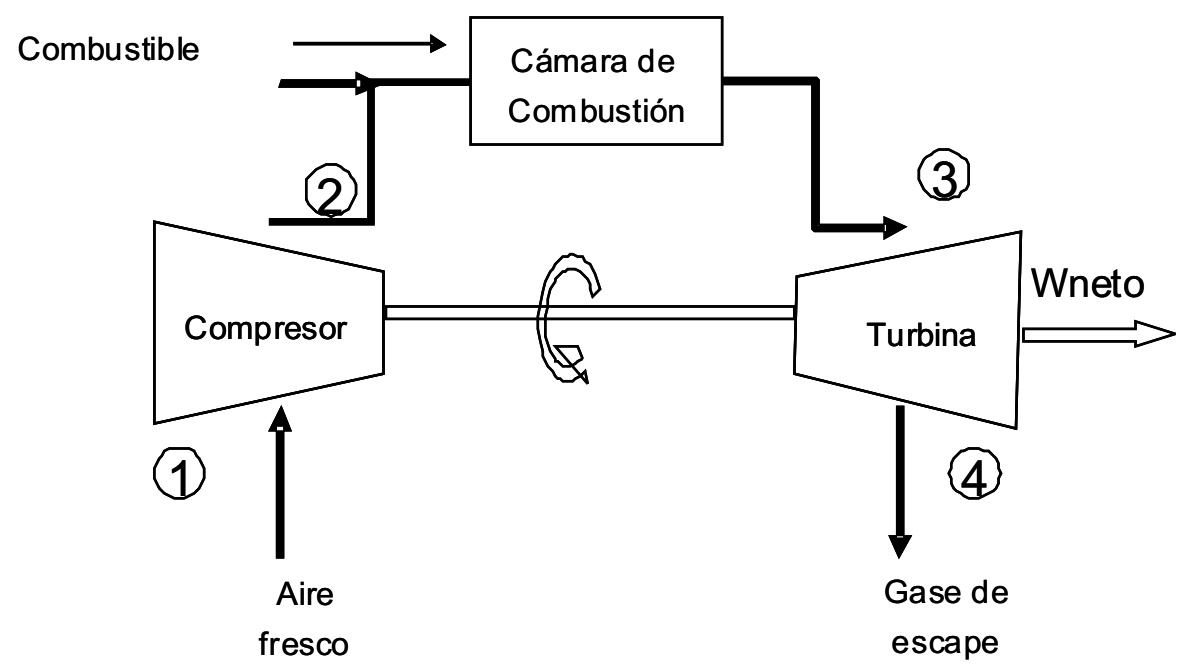

Figura 1. Motor de turbina de gas de ciclo abierto 
Con estas suposiciones el ciclo Brayton (Wark y Richards, 2001) se compone de los siguientes procesos reversibles (figura 2 ):

$$
\begin{aligned}
& 1 \rightarrow 2) \quad \text { Compresión adiabática. } \\
& 2 \rightarrow 3) \quad \text { Calentamiento isobárico. } \\
& 3 \rightarrow 4) \quad \text { Expansión adiabática. } \\
& 4 \rightarrow 1) \quad \text { Enfriamiento isobárico. }
\end{aligned}
$$

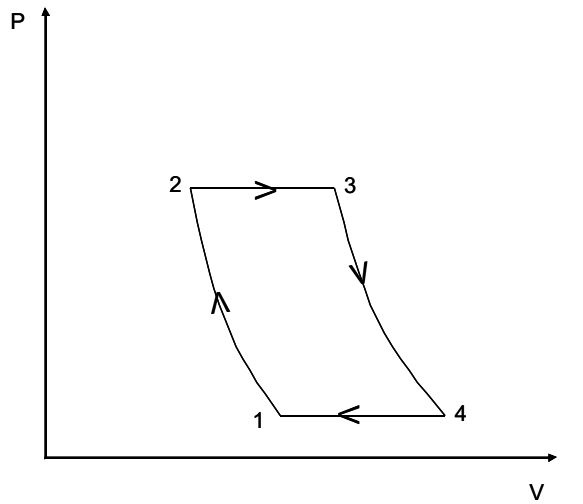

Figura 2. Diagrama $P-V$ de un ciclo Brayton

De acuerdo con las aproximaciones realizadas para un ciclo Brayton y utilizando la convención que define el trabajo durante un proceso reversible como (Wark y Richards, 2001) el trabajo neto o total estará dado por:

$$
\begin{aligned}
& W_{2 \rightarrow 3}=R\left(T_{2}-T_{3}\right) \\
& W_{3 \rightarrow 4}=C_{v}\left(T_{4}-T_{3}\right) \\
& W_{4 \rightarrow 1}=R\left(T_{4}-T_{1}\right)
\end{aligned}
$$$$
W_{\text {neto }}=W_{1 \rightarrow 2}+W_{2 \rightarrow 3}+W_{3 \rightarrow 4}+W_{4 \rightarrow 1}
$$$$
\text { donde } W_{1 \rightarrow 2} \text { es } W_{1 \rightarrow 2}=C_{v}\left(T_{2}-T_{1}\right)
$$

por lo que $W_{\text {neto }}=C_{v}\left(T_{2}-T_{1}\right)+R\left(T_{2}-T_{3}\right)+$

$$
C_{v}\left(T_{4}-T_{3}\right)+R\left(T_{4}-T_{1}\right)
$$

esta expresión se puede anotar de la siguiente manera:

$$
\begin{aligned}
W_{\text {neto }}= & C_{V}\left[\left(T_{4}-T_{1}\right)+\left(T_{2}-T_{3}\right)\right]+ \\
& R\left[\left(T_{4}-T_{1}\right)+\left(T_{2}-T_{3}\right)\right] .
\end{aligned}
$$

Agrupando términos semejantes y extrayendo $T_{1}$ como factor común queda que:

$$
W_{\text {neto }}=C_{p} T_{1}\left[\left(\frac{T_{4}}{T_{1}}-1\right)+\left(\frac{T_{2}}{T_{1}}-\frac{T_{3}}{T_{1}}\right)\right]
$$

En el ciclo Brayton se ha definido la relación de presiones como

$$
r_{p}=\frac{P_{2}}{P_{1}}
$$

y teniendo en cuenta que para el proceso 1-2 se cumple que:

$$
\frac{T_{1}^{C P} / R}{P_{1}}=\frac{T_{2}^{C_{p} / R}}{P_{2}},
$$

se obtiene:

$$
\frac{T_{2}}{T_{1}}=r_{p} \frac{\gamma-1}{\gamma},
$$

donde

$$
\gamma=\frac{C_{p}}{C_{V}}
$$

del mismo modo y tomando en cuenta $P_{1}=P_{4}$ y $P_{2}=P_{3}$ también se demuestra que

$$
\frac{T_{3}}{T_{4}}=r_{p} \frac{\gamma-1}{\gamma}
$$

Reemplazando (10) y (12) en la expresión (8) queda:

$$
W_{\text {neto }}=C_{p} T_{1}\left[\left(\frac{T_{3}}{T_{1}} \boldsymbol{r}_{p} \frac{1-\gamma}{\gamma}-1\right)+\left(\boldsymbol{r}_{p} \frac{y-1}{\gamma}-\frac{T_{3}}{T_{1}}\right)\right]
$$

El trabajo $W_{1 \rightarrow 2}$ y $W_{3 \rightarrow 4}$ se ha definido como el trabajo de entrada al compresor y el trabajo de salida de la turbina, respectivamente, y la relación entre el trabajo del compresor y el trabajo de la turbina

$$
r_{t r}=\frac{W_{1 \rightarrow 2}}{W_{3 \rightarrow 4}}
$$


recibe el nombre de relación del trabajo de retroceso o relación de acoplamiento turbina-compresor (Cengel y Boles, 2006; Wark y Richards, 2001).

Las dos principales áreas de aplicación de las turbinas de gas son la propulsión de aviones y la generación de energía eléctrica (Cengel y Boles, 2006). Cuando se utilizan en propulsión de aviones, la turbina de gas produce suficiente potencia para accionar el compresor y los gases de escape de alta velocidad son los responsables de producir el empuje necesario para impulsar la aeronave (Cengel y Boles, 2006; Burghardt, 1984). Las centrales eléctricas de turbina de gas son empleadas por la industria de generación de energía eléctrica en emergencias, debido a su bajo costo y rápido tiempo de respuesta.

El ciclo de turbina de gas también puede ejecutarse como un ciclo cerrado para ser utilizado en centrales nucleoeléctricas, en la que se emplean como sustancias de trabajo gases como el helio o el argón (Cengel y Boles, 2006). Es importante resaltar el hecho de que en las plantas eléctricas de turbina a gas la relación de trabajo de retroceso suele ser muy alta (Cengel y Boles, 2006; Wark y Richards, 2001), debido a que más de la mitad de la salida de trabajo de la turbina se utiliza para activar el compresor, por lo que una central eléctrica requiere de grandes turbinas para cumplir con los requerimientos de potencia del compresor.

\section{Análisis}

Utilizando el software suministrado por Cengel y Boles (2006) que permite estudiar ciclos de potencia de vapor y de gas de diversas complejidades, así como efectuar estudios de combustión de diversos combustibles en sistemas cerrados y abiertos y evaluar propiedades de mezclas de vapor de aire-agua y de refrigerantes, se analizó la influencia de la relación de presiones, temperatura máxima y temperatura mínima del ciclo en el trabajo neto y en la relación de trabajo de retroceso en un ciclo Brayton.

La temperatura de entrada al compresor o temperatura mínima era de $300 \mathrm{~K}$ y la temperatura máxima o temperatura de entrada a la turbina de $1800 \mathrm{~K}$. La presión al inicio del proceso de compresión era de $100 \mathrm{kPa}$.

El estudio se realizó para tres sustancias de trabajo diferentes, que en este caso fueron Argón, Aire y Oxígeno. Se consideró que las capacidades térmicas se mantienen constantes con la temperatura.

En la tabla 1 se puede observar que el trabajo neto depende de la sustancia de trabajo que se esté utilizando y aumenta en medida que incrementa la relación de trabajo de retroceso, ésta alcanza un máximo y luego empieza a disminuir, lo que también se observa en las figuras 3,4 y 5 .

Es interesante mencionar el hecho de que este máximo no va a depender de la sustancia de trabajo, pues es el mismo para argón, aire y oxigeno, lo que no ocurre con la relación de presiones, la cual también alcanza un punto de trabajo máximo, pero sí va a depender del tipo de fluido que se esté usando (Cengel y Boles, 2006; Wark y Richards, 2001; Jones y Dugan, 1997 y Russel y Adebiyi, 1997).

Tabla 1. Valores de la relación de presiones, trabajo neto y relación de trabajo de retroceso para Argón, Aire y Oxígeno

\begin{tabular}{ccccccccc}
\hline \multicolumn{3}{c}{ Argón $(\gamma=1.667)$} & \multicolumn{3}{c}{ Aire $(\gamma=1.4)$} & \multicolumn{3}{c}{ Oxígeno $(\gamma=1.395)$} \\
\hline $\begin{array}{c}\text { Relación de } \\
\text { presiones }(\mathrm{r}) \\
\mathrm{P}_{2} / \mathrm{P}_{1}\end{array}$ & $\begin{array}{c}\text { Trabajo } \\
\begin{array}{c}\text { Neto Wneto } \\
(\mathrm{KJ} / \mathrm{Kg})\end{array}\end{array}$ & $\begin{array}{c}\text { Relación de } \\
\text { trabajo } \\
\text { retroceso } \\
(\mathrm{Wc} / \mathrm{Wt})\end{array}$ & $\begin{array}{c}\text { Relación de } \\
\text { presiones }(\mathrm{r}) \\
\mathrm{P}_{2} / \mathrm{P}_{1}\end{array}$ & $\begin{array}{c}\text { Trabajo } \\
\begin{array}{c}\text { (eto Wneto } \\
(\mathrm{KJ} / \mathrm{Kg})\end{array}\end{array}$ & $\begin{array}{c}\text { Relación de } \\
\text { trabajo } \\
\text { retroceso } \\
(\mathrm{Wc} / \mathrm{Wt})\end{array}$ & $\begin{array}{c}\text { Relación de } \\
\text { presiones }(\mathrm{r}) \\
\mathrm{P}_{2} / \mathrm{P}_{1}\end{array}$ & $\begin{array}{c}\text { Trabajo } \\
\text { Neto Wneto } \\
(\mathrm{KJ} / \mathrm{Kg})\end{array}$ & $\begin{array}{c}\text { Relación de } \\
\text { trabajo de } \\
\text { retroceso } \\
(\%)\end{array}$ \\
\hline 4 & 283 & 29.02 & 19 & 631.1 & 38.65 & 18 & 574.7 & 37.76 \\
5 & 303.5 & 31.73 & 20 & 632.1 & 39.22 & 19 & 576.2 & 38.34 \\
6 & 315.7 & 34.13 & 20 & 632.8 & 39.77 & 20 & 577.3 & 38.90 \\
7 & 322.7 & 36.30 & 21 & 633.2 & 40.30 & 21 & 578.1 & 39.44 \\
8 & 326.4 & 38.29 & 22 & 633.3 & 40.82 & 22 & 578.6 & 39.96 \\
9 & 327.9 & 40.14 & 23 & 633.2 & 41.82 & 23 & 578.8 & 40.47 \\
10 & 327.7 & 41.86 & 24 & 632.9 & 41.80 & 24 & 578.9 & 40.96 \\
11 & 326.7 & 43.49 & 25 & 632.4 & 42.27 & 25 & 578.7 & 41.43 \\
12 & 324.3 & 45.03 & 26 & 631.8 & 42.73 & 26 & 578.4 & 41.90 \\
\hline
\end{tabular}


DOI: http://dx.doi.org/10.22201/fi.25940732e.2010.11n3.022

Malaver de la Fuente $\mathrm{M}$.

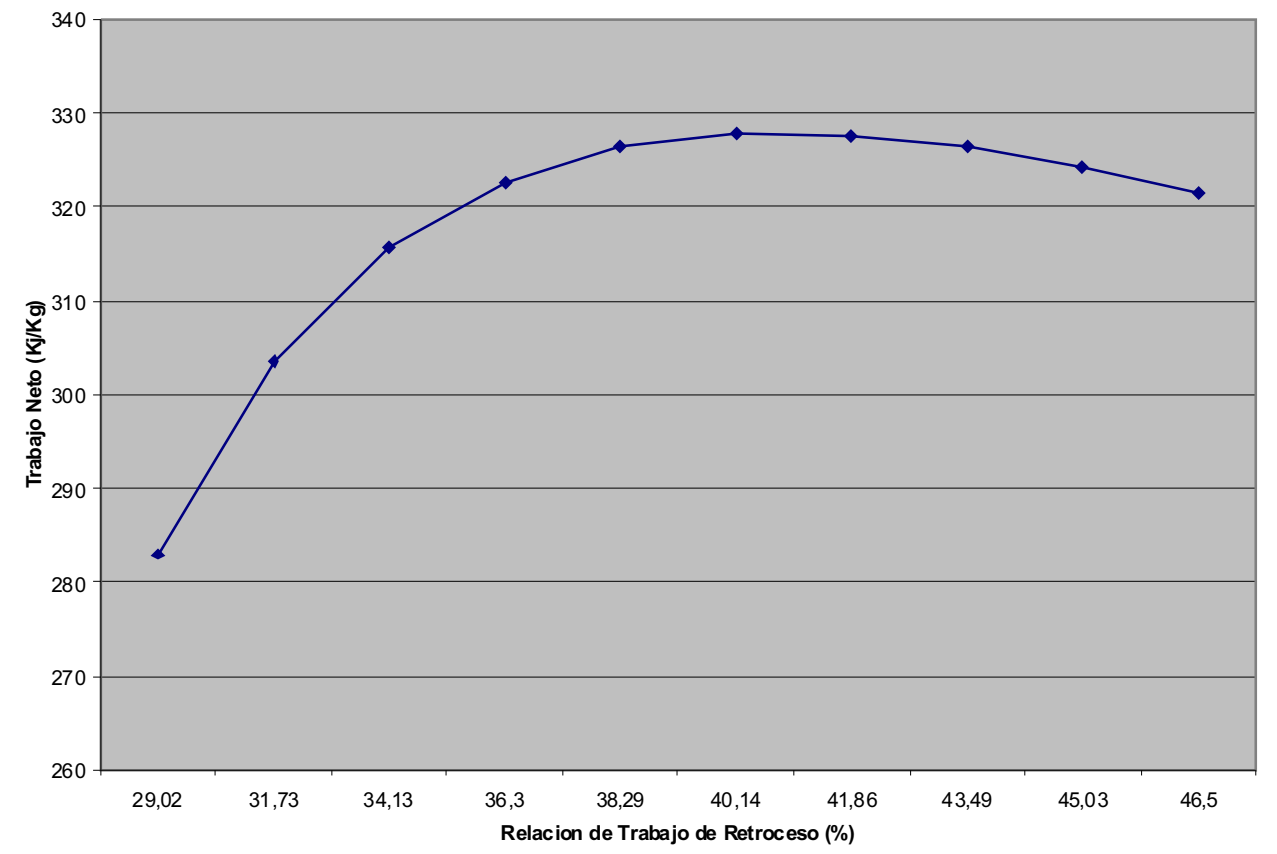

Figura 3. Variación del trabajo neto en $\mathrm{kj} / \mathrm{kg}$ versus la relación de trabajo de retroceso para el argón

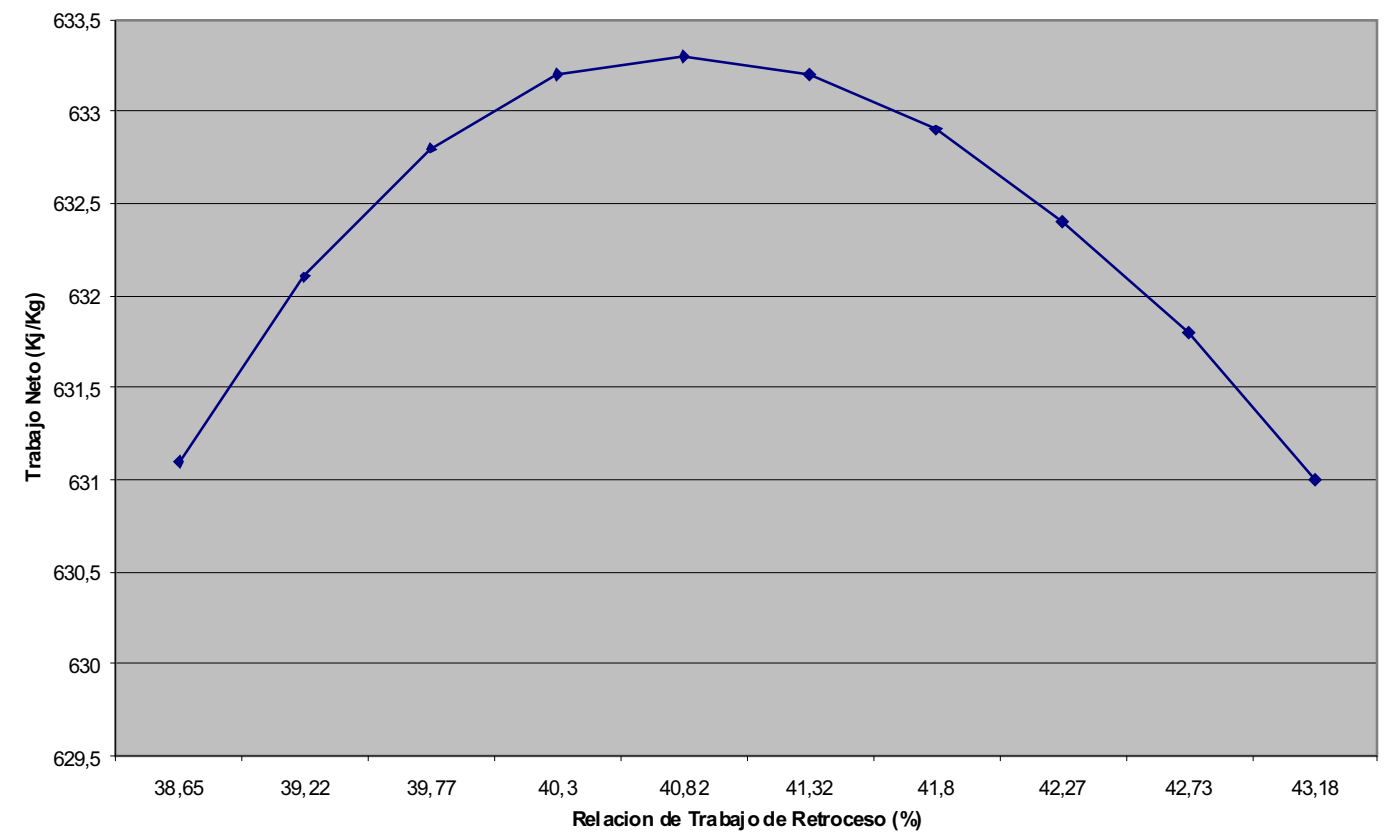

Figura 4. Variación del trabajo neto en kj/kg versus la relación de trabajo de retroceso para el aire 


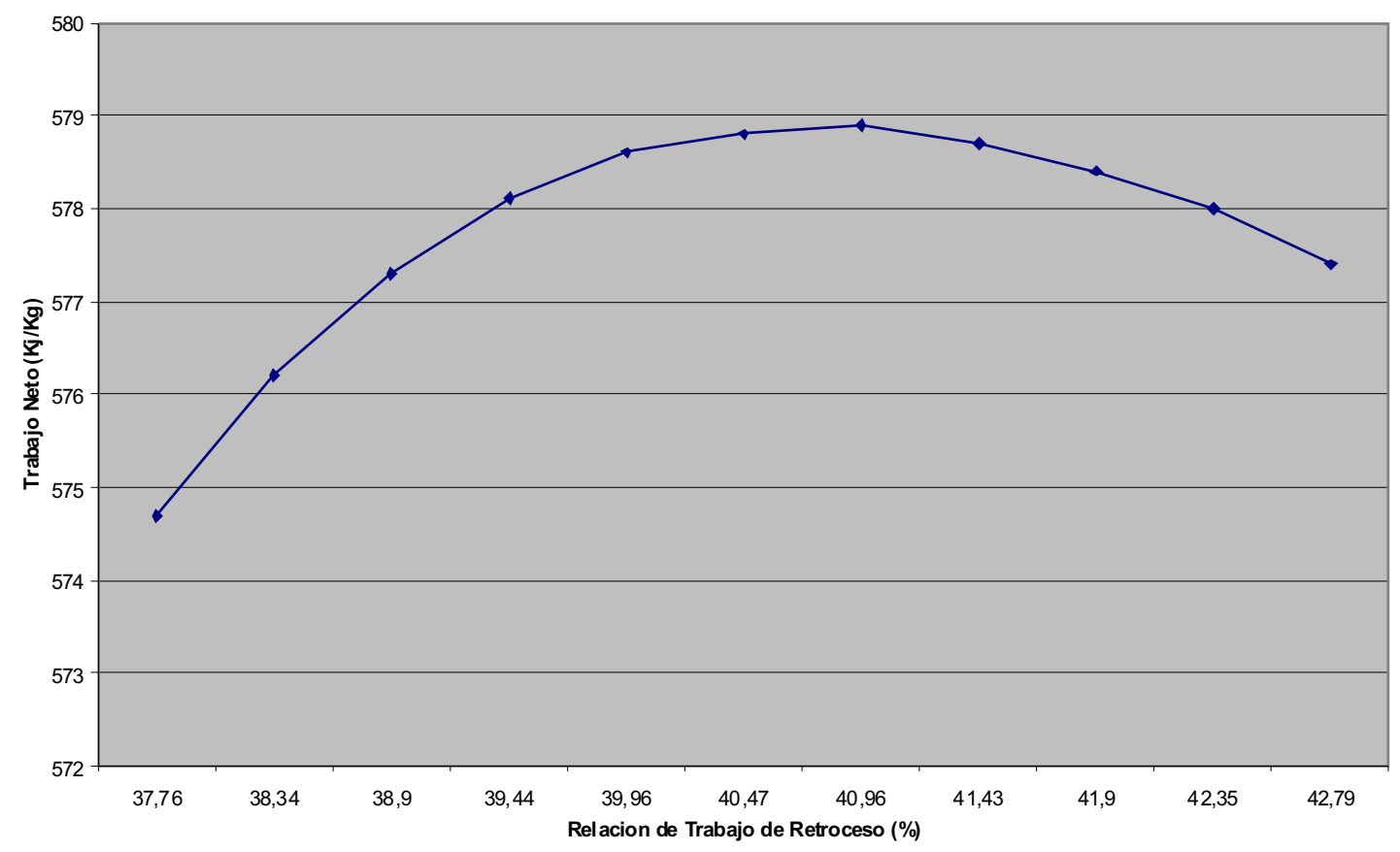

Figura 5. Variación del trabajo neto en $\mathrm{kj} / \mathrm{kg}$ versus la relación de trabajo de retroceso para el oxígeno

Se observa también una dependencia de la relación de acoplamiento con la relación de presiones. Basándose en estos resultados, se puede deducir una expresión para la relación de trabajo de retroceso en función de la sustancia de trabajo y de la relación de presiones. De acuerdo a la definición de

$$
\begin{aligned}
& r_{t r}=\frac{W_{1 \rightarrow 2}}{W_{3 \rightarrow 4}} \text { se tiene que: } \\
& \frac{W_{1 \rightarrow 2}}{W_{3 \rightarrow 4}}=\frac{C_{v}\left(T_{2}-T_{1}\right)}{C_{v}\left(T_{4}-T_{3}\right)}=\frac{T_{1}\left(\frac{T_{2}}{T_{1}}-1\right)}{T_{3}\left(\frac{T_{4}}{T_{3}}-1\right)} .
\end{aligned}
$$

Sustituyendo (10) y (12) en (14) se obtiene:

$$
r_{t r}=\frac{W_{1 \rightarrow 2}}{W_{3 \rightarrow 4}}=\frac{T_{1}\left(r_{p} \frac{\gamma-1}{\gamma}-1\right)}{T_{3}\left(r_{p} \frac{1-\gamma}{\gamma}-1\right)} .
$$

Por lo que la ecuación (15) se puede expresar como:

$$
r_{t r}=-r_{p} \frac{\gamma-1}{\gamma} \frac{T_{1}}{T_{3}}
$$

De acuerdo con (16) la relación de trabajo de retroceso para un ciclo de Brayton ideal es función de la relación de presiones, de la sustancia de trabajo, la temperatura de entrada al compresor $T_{1}$ y temperatura de entrada a la turbina $T_{3}$.

Utilizando el procedimiento sugerido en Malaver (2008) y Urrecheaga y Malaver (2003), la expresión (16) se puede sustituir en (13) con el fin de determinar una relación de trabajo de retroceso óptima para que un motor de turbina a gas suministre un trabajo máximo.

En efecto, sustituyendo (16) en (13) la expresión $W_{\text {total }}$ se puede escribir como:

$$
W_{\text {neto }}=-C_{p} T_{1}\left[\frac{1}{r_{t r}}+\frac{T_{3}}{T_{1}}\left(1+r_{t r}\right)+1\right]
$$

El trabajo neto en un ciclo Brayton ideal es función de la relación de trabajo de retroceso y de las temperaturas máxima y mínimas del ciclo, a diferencia de la expresión propuesta por Guzmán-Vargas y Angulo-Brown 
(1998) en la que el trabajo es función de las temperaturas del ciclo y de los procesos $2 \rightarrow 3$ y $4 \rightarrow 1$ (figura 2) y no se muestra la dependencia con la relación de trabajo de retroceso.

En el punto de trabajo máximo se tiene que:

$$
\frac{d W_{n e t o}}{d r_{t r}}=C_{p} T_{1}\left[-\frac{1}{r_{t r}^{2}}+\frac{T_{3}}{T_{1}}\right]=0
$$

por lo que

$$
r_{t r \max }=\sqrt{\frac{T_{1}}{T_{3}}}
$$

Cengel y Boles (2006), Van Wylen et al. (2004) Wark y Richards (2001) definen la eficiencia térmica del ciclo Brayton ideal como:

$$
\eta=1-\frac{1}{r_{p} \frac{\gamma-1}{\gamma}}
$$

Sustituyendo la ecuación (16) en (20) se obtiene para la eficiencia térmica:

$$
\eta=1-\frac{T_{1}}{T_{3} r_{t r}}
$$

Reemplazando (19) en (21) en el punto de trabajo máximo la eficiencia estará dada por:

$$
\eta_{t r \max }=1-\sqrt{\frac{T_{1}}{T_{3}}}
$$

Se deduce entonces, que la eficiencia térmica para un ciclo Brayton ideal en el punto de trabajo óptimo es función de la temperatura de entrada al compresor y de la temperatura de entrada a la turbina. La ecuación (22) es análoga a la expresión encontrada por Leff (1987) en el estudio de la eficiencia del ciclo Otto.

De acuerdo con (19) para un ciclo Brayton ideal la relación de trabajo de retroceso máxima $r_{\text {trmáx }}$ sólo dependerá de las temperaturas máximas y mínimas del ciclo, por lo que es independiente de la sustancia de trabajo. En las figuras 3, 4 y 5 se observa que el trabajo neto aumenta con la relación de trabajo de retroceso, alcanza un máximo y luego disminuye.
El valor máximo que alcanza la relación de acoplamiento turbina-compresor siempre es la misma aún cuando sea diferente la sustancia de trabajo utilizada, lo que concuerda con la expresión (19). Los textos universitarios de termodinámica no mencionan este comportamiento (Cengel y Boles, 2006; Wark y Richards, 2001; Van Wylen et al., 2000; Jones y Dugan, 1997 y Russel y Adebiyi, 1997), son pocos los que hacen una breve referencia a la relación de trabajo de retroceso o relación de acoplamiento en el estudio de los ciclos de potencia de gases como lo hacen Cengel y Boles (2006) y Wark y Richards (2001).

\section{Conclusiones}

El software utilizado es capaz de predecir las condiciones de operación que permiten obtener una relación de acoplamiento máxima en un ciclo Brayton.

Aun cuando los valores de $r_{t r}$ son función del coeficiente $\gamma$ de la sustancia de trabajo, de las temperaturas de entrada al compresor y a la turbina y de la relación de presiones $r_{p}$, el valor máximo de $r_{t r}$ se mantiene constante siempre que $T_{1}$ y $T_{3}$ se mantengan fijos.

Los valores de la relación de presiones para los que se alcanza el trabajo máximo varían dependiendo de la sustancia de trabajo utilizada y aumentan a medida que crece el coeficiente $\gamma$ para $T_{1}$ y $T_{3}$ fijos. Para un ciclo Brayton ideal, altos valores de la relación:

$$
\frac{T_{1}}{T_{3}}
$$

incrementan la relación de trabajo de retroceso máxima.

\section{Agradecimientos}

Se agradece a la Profesora Lucia Santeramo del Centro Bolivariano de Informática y Telemática y de la Universidad Marítima del Caribe por su valioso apoyo en la elaboración de los diagramas y figuras de este artículo.

\section{Referencias}

Burghardt M.D. Ingeniería Termodinámica. México, DF. Editorial Harla. 1984.

Cengel Y. y Boles M. Termodinámica. México, DF. McGrawHill Interamericana. 2006.

Curzon F.L y Ahlborn B. Efficiency of a Carnot Engine at Maximum Power Output. Am.J.Phys, 43(22):22-24. 1975. 
DOI: http://dx.doi.org/10.22201/fi.25940732e.2010.11n3.022

La relación de trabajo de retroceso de un ciclo Brayton

Guzmán-Vargas L. y Angulo-Brown F. A Dissipative Joule-Brayton Cycle Model. Revista Mex. Fís, 44(6): 619-623. 1998.

Herrera C.A, Rosillo M.E y Castaño L. Fugas de calor y aprovechamiento de efluentes en la optimización de ciclos Brayton totalmente irreversibles. Revista Mex. Fís, 54(2): 118 126. 2008.

Jones J.B. y Dugan R.E. Ingeniería Termodinámica. México, DF. Prentice-Hall Hispanoamérica, S.A. 1997.

Leff H. Thermal Efficiency Work Output: New Results for Old Heat Engines. Am.J.Phys, 55(7):602-610. 1987.
Malaver M. Análisis de las temperaturas, la eficiencia térmica y el trabajo neto en un ciclo dual. Educ. Quím, 19(2): 122-126. 2008.

Russel L.D. y Adebiyi G.A. Termodinámica Clásica. Delaware, E.U.A. Addison-Wesley Iberoamericana, S.A. 1997.

Urrecheaga K. y Malaver M. Análisis de las temperaturas y de la eficiencia térmica en el ciclo Otto. Educ. Quim, 14(3): 138-141. 2003.

Van Wylen G.J., Sonntag R.E. y Borgnakke C. Fundamentos de Termodinámica. México, DF. Editorial Limusa, S.A. 2000.

Wark K.J. y Richards D. Termodinámica. Madrid. McGraw-Hill Interamericana, S.A. 2001.

\section{Semblanza del autor}

Manuel Malaver de la Fuente. Licenciado en química egresado de la Universidad Central de Venezuela (UCV). Magíster en educación en la Universidad Pedagógica Experimental Libertador. Candidato a doctor en mecánica teórica y aplicada en la Facultad de Ingeniería de la UCV. Miembro del programa de promoción del investigador desde 2007 a la fecha en la categoría de investigador nivel I. Profesor de la Universidad Marítima del Caribe desde enero de 2007 en el área de termodinámica, cálculo numérico y física general. Profesor del área de hidráulica y mecánica de fluidos en la Universidad Alejandro de Humboltd, desde enero de 2003 a diciembre de 2006. 